\title{
Pratiques
}

Linguistique, littérature, didactique

165-166 | 2015

Étudier les figures en contexte : quels enjeux ?

\section{Les figures de construction et la création d'une syntaxe des accords complexes dans l'histoire de la grammaire latine}

Figures of Syntax and the Development of a Syntax of Complex Agreement in the History of Latin Grammar

\section{Bernard Colombat}

\section{OpenEdition}

Journals

Édition électronique

URL : http://journals.openedition.org/pratiques/2471

DOI : $10.4000 /$ pratiques. 2471

ISSN : 2425-2042

Éditeur

Centre de recherche sur les médiations (CREM)

\section{Référence électronique}

Bernard Colombat, «Les figures de construction et la création d'une syntaxe des accords complexes dans l'histoire de la grammaire latine », Pratiques [En ligne], 165-166 | 2015, mis en ligne le 01 octobre 2015, consulté le 20 avril 2019. URL : http://journals.openedition.org/pratiques/2471 ; DOI : 10.4000/ pratiques. 2471

Ce document a été généré automatiquement le 20 avril 2019

(c) Tous droits réservés 


\section{Les figures de construction et la création d'une syntaxe des accords complexes dans l'histoire de la grammaire latine}

Figures of Syntax and the Development of a Syntax of Complex Agreement in the History of Latin Grammar

\section{Bernard Colombat}

1 L'interrogation initiale est la suivante: dans les textes grammaticaux humanistes, il y a des termes, différenciés, assez nombreux, pour désigner ce qu'on appelle, depuis le Moyen Âge, des «figures de construction ", mais les définitions varient, et les exemples utilisés sont parfois différents, voire contradictoires. Néanmoins, du fait: (1) que les grammairiens du latin au début $\mathrm{du} \mathrm{XVI}^{\mathrm{e}}$ siècle s'accordent à reconnaitre, de façon relativement stable, huit figures de construction, (2) qu'ils les mettent sur un même pied d'égalité, (3) que ces figures sont conçues comme fonctionnant en réseau, l'objet de cet article est de voir l'état de ce réseau et de tenter d'en expliquer les fondements historiques. Le schéma évoluera (les huit figures seront réduites à quatre figures principales à la fin $\mathrm{du} \mathrm{XVI}^{\mathrm{e}}$ siècle) et nous ne pourrons pas retracer le détail de cette évolution (sur ce point, $c f$. Colombat, 1993). Il s'agira surtout ici de voir comment ce concept de "figure de construction » a pu se créer et se développer, avant de se diluer dans la syntaxe latine d'accord.

\section{Deux textes comme point de départ: Donat, Ars maior et Scaliger, De causis}

On partira de deux textes, l'un et l'autre aux deux extrémités de la période examinée. Le premier est celui de Donat. 
[1] Donat, Ars maior, livre 3, § 5, De schematibus, 663.13-664.7 H.

Zeugma est unius uerbi conclusio diuersis clausulis apte coniuncta, ut

[TRO $\left.{ }^{1}\right]$ Troiugena interpres diuum, qui numina Phoebi,

qui tripodas, Clari laurus, qui sidera sentis

et cetera.

Hypozeuxis est figura superiori contraria, ubi diuersa uerba singulis clausulis

subiunguntur, ut

regem adit et regi memorat nomenque genusque

et cetera

Syllempsis est dissimilium clausularum per unum uerbum conglutinata conceptio, ut

[HIC] hic illius arma, / hic currus fuit.

Hoc schema ita late patet, ut fieri soleat et per partes orationis, et per accidentia partibus orationis.

«Le zeugme est l'action d'enfermer, par association correcte, un seul mot dans divers syntagmes, par ex.

[TRO] Fils de Troie, interprète des dieux, toi qui perçois les volontés de Phébus,

le trépied prophétique, le laurier de l'hôte de Claros, les étoiles, etc.

L'hypozeuxis est la figure contraire à la précédente, dans laquelle différents mots sont rattachés à chaque syntagme, par ex.

Il va voir le roi et il rappelle au roi son nom, et sa race, etc.

La syllepse est l'action de contenir, par agglutination, des syntagmes hétérogènes par l'intermédiaire d'un seul mot, par ex.

[HIC] Là furent ses armes, là son char.

Cette figure a une extension si large qu'elle se produit d'ordinaire à la fois par les parties du discours et par les accidents des parties du discours ». ${ }^{2}$

3 Ces figures ne sont pas, par elles-mêmes, syntaxiques, c'est-à-dire qu'elles n'ont pas d'incidence en morphosyntaxe: dans TRO, mettre un verbe en facteur commun avec plusieurs compléments (zeugme) n'a pas, dans une langue comme le latin, d'incidence syntaxique. Répéter regem sous la forme regi (hypozeuxis) n'a pas plus d'incidence sur ce plan.

4 En revanche, elles peuvent en avoir : dans HIC, on a le sentiment qu'il manque quelque chose, que sous fuit, il y a une forme qui serait fuerunt. Et si Donat insiste sur la grande extension de cette figure, faisant intervenir des parties du discours et des accidents, c'est certainement parce qu'il a conscience qu'elle peut être fauteuse de troubles.

5 Le deuxième texte est pris à l'autre bout de la période. Il s'agit du chapitre $179 \mathrm{du} D e$ causis linguae Latinae de Jules-César Scaliger (1540), chapitre consacré à la conceptio et qui se trouve dans le livre 12, lui-même consacré à la figure.

[2] Scaliger. De causis, 1540, livre 12, la figure, chap. 179, Conceptio (syllepse), p. 336.

Quemadmodum una fieret oratio in superiore libro et alibi dictum est, coniunctione enim fit una: Caesar et Cato equitant. 'Equitatio' hic una est in duobus. Itaque aliquando subiecta intelligis, quae quia sunt plura, pluralem numerum appones; aliquando praedicati unitatem communem utrique accipies, quare numerum attribues unitatis, Caesar et Cato equitat. Maluerunt igitur illam esse figuram in plurali, quam hanc in singulari. Et ratio est quia coniunctio repetit numerum singularem : ostendimus enim duas esse orationes potestate, quare utraque erit singularis [...].

Paulo figuratius eadem oratio in obliquis versatur, sic: Caesar cum Catone disputant. Cuius loquutionis necessitas evenit ad evitandam ambiguitatem. Si diceres Caesar cum Catone disputat, non unionem sed controversiam posses accipere. Sed illa longe figuratior apud Ovidium : 
[ILI] Ilia cum Lauso de Numitore sati.

Neque enim Ilia erat nati, sed nata cum Lauso. Itaque ante quam reddas verbum recto, rectus cum obliquo ita sunt coniungendi, ut unum sit e pluribus, quibus pluribus verbi numerus respondeat. Recte vero putarunt illam esse figuram apud Poëtam :

[REM] Cana fides et Vesta, Remo cum fratre Quirinus iura dabunt.

Sed nos etiam utroque modo figuram intelligimus : Fides et Vesta iura dabunt, et alteram in obliquo, Quirinus cum Remo.

«Au livre précédent et ailleurs, il a été dit de quelle façon l'énoncé s'unifie, et c'est au moyen de la conjonction: "César et Caton vont à cheval (equitant)." Le fait d'aller à cheval (equitatio) est en l'occurrence une seule action en deux personnes. C'est ainsi que, parfois, tu penses aux sujets, et comme ils sont plusieurs, tu leur apposeras un pluriel; parfois tu considéreras l'unité du prédicat commun à tous deux, et dès lors tu leur attribueras le nombre de l'unité : "César et Caton [litt.] va à cheval (equitat)." Ainsi donc on a préféré qu'il y ait cette figure dans le cas de l'énoncé au pluriel plutôt qu'une autre dans le cas de l'énoncé au singulier. Et la raison, c'est que la conjonction réclame le singulier. Nous avons montré en effet qu'il y a en puissance deux énoncés: dès lors, chacun des deux sera au singulier [...].

De manière un peu plus figurée, le même énoncé fraye avec les cas obliques : "César [litt.] avec Caton discutent (cum Catone disputant)". C'est afin d'échapper à l'ambiguïté que ce tour a surgi comme indispensable. Si tu disais : "César avec Caton discute (disputat)", tu pourrais considérer qu'il s'agit, non pas d'union, mais de litige. Mais en voici un bien davantage figuré chez Ovide :

[ILI] Ilia cum Lauso de Numitore sati. "Ilia avec Lausus engendrés par Numitor."

Et de fait Ilia ne fut pas sati (engendrés) avec Lausus, mais sata (engendrée). Par conséquent, avant que tu ne rapportes le verbe au cas direct [nomin.], le direct et l'oblique sont à conjoindre de telle sorte que l'on ait une unité issue d'une pluralité, pluralité à laquelle réponde le nombre du verbe. Et certes on a eu raison de penser que c'était cette figure qu'on a chez le Poète :

[REM] Cana fides et Vesta, Remo cum fratre Quirinus iura dabunt. "La blanche Foi et Vesta, Quirinus avec son frère Rémus donneront des lois."

Mais pour notre part, nous entendons même qu'il y a figure de deux façons :

"La Foi et Vesta donneront des lois" et - seconde figure dans l'oblique :

"Quirinus avec Rémus".»

6

paraitre étonnant à un moderne. Mais si l'on accepte, comme Scaliger, qu'une conjonction conjoint non pas deux mots, mais deux énoncés, on peut admettre que c'est le second énoncé le plus normal des deux, car est maintenue la forme du verbe telle qu'elle apparait dans chacun des deux énoncés. Retenons aussi le principe d'une gradation: dans l'exemple suivant ILI, il y a une figure plus complexe, parce que Ilia [féminin] est contenu dans le masculin pluriel [sati], mais aussi, ce que ne dit pas Scaliger, parce que l'accord au pluriel se fait avec un nominatif (Ilia) accordé avec un nom à l'ablatif précédé de cum (ce qu'on appelle alors syllepse par cum).

Et le dernier exemple REM est encore plus intéressant : Scaliger y voit une double figure, la première par association du pluriel dabunt avec un double sujet au singulier, la seconde par l'association d'un nominatif avec un ablatif. Mais cette association de trois nominatifs singuliers avec un verbe au pluriel n'a pour nous plus rien d'étonnant. Elle est même au contraire de la plus grande banalité. Il est à noter cependant que l'exemple est parfois 
donné sous la forme du deuxième syntagme seulement: Remo cum fratre Quirinus iura dabunt ; il devient alors beaucoup plus intéressant syntaxiquement, puisqu'il présente un seul nominatif associé avec un ablatif, cas typique de syllepse par cum.

\section{Retour à Priscien, Ars, 17, § 155}

Retour à ce qui est pour nous le point de départ : un court passage du livre 17 de Priscien crucial dans l'histoire de la syntaxe occidentale, car il a servi à bâtir tout un arsenal de figures dites « de construction » dont le déploiement a permis de traiter des phénomènes complexes d'accord.

[3] Priscien, Ars [Institutiones grammaticae], livre 17, § 155, GL 3, 183.17-184.5

Variantur autem, ut dictum est, per transitiones et reciprocationes non solum casus et numeri, sed etiam genera, ut illos laedit mulier et illum laedunt mulieres. Superuacuum est in re tam manifesta usus auctorum testimonia proferre; illud tamen sciendum quod per figuram quam Graeci $\alpha \lambda \lambda$ oló $\eta \tau \alpha$ uocant, id est uariationem,



et per $\zeta \varepsilon \tilde{v} \gamma \mu \alpha$, id est adiunctionem

et concidentiam, quam $\sigma u v \varepsilon ́ \mu \pi \tau \omega \sigma \mathrm{v} v$ Graeci uocant, uel procidentiam, id est $\dot{\alpha} v \tau i ́ \pi \tau \omega \sigma \downarrow v$, et numeri diuersi et diuersa genera et diuersi casus et tempora et personae non solum transitiue et per reciprocationem, sed etiam intransitiue copulantur, quae diuersis auctorum exemplis tam nostrorum quam Graecorum necessarium esse duximus comprobare.

"À côté de cela, il y a des variations, comme on l'a dit, non seulement de cas et de nombres, mais aussi de genres, du fait de la transition ou de la réflexion, comme dans illos laedit mulier [une femme offense ceux-ci], illum laedunt mulieres [des femmes offensent celui-ci] : il est superflu sur un sujet aussi évident de citer le témoignage de l'usage des auteurs. Ce qu'il faut savoir en revanche, c'est que la figure que les Grecs appellent alloiotês, c'està-dire la variation, que ce soit :

- la prolêpsis ou la sullêpsis, c'est-à-dire l'anticipation ou l'englobement,

- le zeugma, c'est-à-dire l'adjonction,

- la coïncidence, que les Grecs appellent sunemptôsis, ou la mutation casuelle, c'est-à-dire l'antiptôsis,

fait que des nombres différents, des genres différents, et des cas, des temps et des personnes différents se trouvent associés non seulement quand il y a transition ou réflexion, mais même intransitivement. Cela, nous avons pensé qu'il était nécessaire de le confirmer par différents exemples empruntés aux auteurs, aussi bien latins que grecs » (trad. Groupe Ars grammatica, 2010 : 241-242).

Partons du début : la remarque concernant la variatio observable dans illos laedit mulier [une femme offense ceux-ci], illum laedunt mulieres [des femmes offensent celui-ci] est pour nous étrange : il est bien évident qu'un sujet singulier peut avoir des objets au pluriel, et inversement ; mais ce qui est remarquable, c'est que Priscien éprouve le besoin d'en faire mention, même s'il ajoute qu'« il est superflu sur un sujet aussi évident de citer le témoignage de l'usage des auteurs».

Suit une liste de termes grecs, avec à chaque fois des doublets latins :

- $\dot{\alpha} \lambda \lambda$ oı́́ $\eta \tau \alpha=$ uariationem

- $\pi \rho{ }^{\prime} \lambda \eta \psi \mathrm{iv}=$ praeceptionem

- $\sigma u ́ \lambda \lambda \eta \psi \imath \mathrm{v}=$ conceptionem 
- $\zeta \tilde{\varepsilon} \gamma \mu \alpha=$ adiunctionem

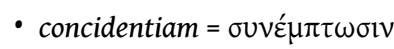

- procidentiam $=\dot{\alpha} v \tau i ́ \pi \tau \omega \sigma \mathrm{lv}$ grec passant généralement en latin, au prix d'une légère adaptation. Un terme latin sera néanmoins souvent employé : conceptio, souvent donné comme équivalent de syllepsis (cf. supra, le texte [2] de Scaliger). et les cinq autres apparaissent comme des espèces de cette uariatio. Le jeu des et et des uel organise ces cinq autres figures sous la forme $2+1+2$ (cf. la présentation du texte dans l'édition du Groupe Ars grammatica, $2010: 243$, note 306 et reproduite ici). masse de son Ars de ces figures: ses expressions favorites, très fréquentes, sont génériques : per figuram ou figurate (de façon figurée). Car ce qui intéresse Priscien, c'est, plus que le détail, le principe de la figure qui permet à la langue de fonctionner, selon une conception de la figure que j'avais qualifiée de «banalisée » et que M Baratin (2011:62) préfère appeler «métalinguistique », un terme au choix duquel j'adhère complètement. C'est le Moyen Âge qui va élaborer un système complexe dont la Renaissance hérite.

\section{Le dispositif en 1500}

Revenons au début du XVI e siècle. En 1500, il y a 8 figures dites de construction :

[4] prolepsis, syllepsis, zeugma, synthesis [synemptosis], antiptosis, euocatio, appositio, synecdoche

On va évoquer, rapidement le sort des plus intéressantes d'entre elles, celles qui entrent dans un réseau, pour rendre saillantes leur fonction et leur évolution.

Il faut remarquer d'abord la disparition du terme désignant la figure générique chez Priscien: l'alloiotês. Citée dans ce texte à l'accusatif, alloiotêta, cette forme a été mal analysée par les médiévaux, qui ont pris cette forme d'accusatif pour une forme de nominatif et qui, sans doute pour «faire plus grec", ont ajouté un $h$ après le $t$, ce qui a donné allotheta, -ae. Le terme a toujours la même fonction de figure générique dans le Catholicon de Giovanni Balbi.

17 Se sont maintenues les figures suivantes: prolepsis, syllepsis, zeugma, antiptosis. À la différence de l'alloiotês, la sunemptosis a subsisté, mais le terme qui la désigne est devenu synthesis, par réduction d'une syllabe et adjonction d'une aspiration.

Trois nouvelles figures apparaissent : deux avec des noms latins (euocatio, appositio), l'une avec un nom grec: la synecdoche. Dans la mesure où elles fonctionnent moins en réseau, nous n'évoquerons que rapidement leur fonction:

- l'évocation tire son origine également du texte de Priscien, qui pose qu'un nom au nominatif est de la $3^{\mathrm{e}}$ personne. Elle décrit les cas où un pronom de $1^{\text {re }}$ personne "appelle " à sa personne le nom qui lui est apposé (ex. ego Priscianus scribo);

- l'apposition diffère de l'évocation en ce qu'il n'y a pas de conflit de personne : les auteurs hésitent entre une définition sémantique (servir à restreindre la généralité, lever une équivoque, attribuer une propriété) et son utilisation pour montrer des éléments apposés hétérogènes du point de vue des accidents (ex. urbs [sg.] Athenae [pl.]) ; 
- la synecdoque (la partie pour le tout, $c f$. aussi Priscien, 18, 27-28, qui décrit le phénomène, mais le désigne simplement par figurate, GL 220.11) sert à traiter les cas de construction où un élément (adjectif ou participe), qui ne s'applique qu'à une partie, est accordé avec l'ensemble, la partie étant à l'accusatif (ex. Aeneas... os humerosque [acc.] deo similis, « Énée... semblable à un dieu par le visage et les épaules »).

\section{La prolepse}

\section{Chez Donat :}

[5] Donat, Ars maior, livre 3, § 5, De schematibus, 663.10-12 H. Prolempsis est praesumptio rerum ordine secuturarum, ut [CON] Continuo reges ingenti mole Latinus et cetera.

«La prolepse est la prise par avance des choses qui, selon l'ordre, doivent suivre, comme

[CON] Immédiatement les rois, Latinus à la taille puissante, etc. »

Cité tel quel, l'exemple est trop bref pour avoir une quelconque incidence en morphosyntaxe: un pluriel, reges, est repris par un singulier, Latinus. Si l'on prend l'exemple en entier, des sujets au singulier suivent, accompagnés chacun d'un verbe au singulier (vehitur, it), avant que n'arrivent deux sujets au singulier coordonnés (hinc pater Aeneas..., et iuxta Ascanius) suivis par un verbe au pluriel: procedunt castris. Deux constructions sont possibles : procedunt peut être ramené aux deux derniers sujets, mais il peut l'être aussi à reges, huit vers plus haut, ce qui impose de considérer tout ce qu'il y a entre comme une incise ( $c f$. notre ponctuation dans la traduction de l'exemple). D'où une ambigüité.

1 Rien de tel chez Priscien, où la prolepse est une des rares figures à être évoquées au moyen d'un terme spécifique avant le passage précité, au $\$ 28$ sq. du livre 17: kata prolepsin et où elle fait l'objet d'une analyse précise :

[6] Priscien, Ars, XVII, 28-29, GL 125.14-126.20

Et sciendum quod in huiuscemodi constructione diuiduorum, si per

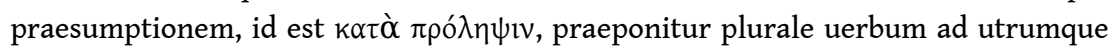
sequens, licet nominatiuum anteferre, ut

[AQVa] aquilae deuolauerunt, haec ab oriente, illa ab occidente ;

sin ad ea quae diuiduntur, singulariter uerba consequentur, genetiuum oportebit praeponi pluralem diuidendum, ut

$[\mathrm{AQVb}]$ aquilarum altera deuolauit ab oriente, altera ab occidente ;

nec mirum hoc fieri, cum uerbum siue praepositum siue consequens iure sibi exigat nominatiuum. [...]

Auctoritas tamen ueterum est, quando pro genetiuo plurali nominatiuum praeponit, quamuis ad sequentes res, id est diuisas, singulariter uerba redduntur, ut Homerus :

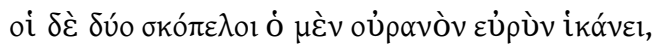

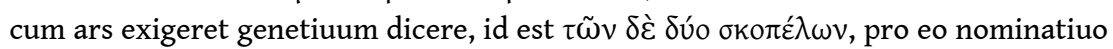
est usus. Similiter si quis dicat

duo fratres, alter maior, alter minor est, uitium facit, debet enim dicere

duorum fratrum alter maior, alter minor est

et :

duorum oculorum alter dexter, alter sinister est,

non:

duo oculi alter dexter, alter sinister est. 
Liuius in XXXXI :

[QAN] periti religionum iurisque publici, quando duo ordinarii consules eius anni, alter morbo, alter ferro periisset, suffectum consulem negabant recte comitia habere posse.

«Il faut savoir en outre que dans ce genre de construction répartitive, si par prolepse, c'est-à-dire kata prolêpsin, le verbe au pluriel est pré-posé par rapport à l'un et l'autre des deux éléments subséquents, on peut mettre d'abord un nominatif, comme dans

[AQVa] aquilae deuolauerunt, haec ab oriente, illa ab occidente [les aigles descendirent du ciel, l'un depuis le levant, l'autre depuis le couchant] ;

si au contraire, par rapport aux éléments répartis, ce sont des verbes au singulier qui suivent, il conviendra alors qu'on pré-pose le génitif pluriel qui fait l'objet de la répartition, comme dans

$[\mathrm{AQVb}]$ aquilarum altera deuolauit ab oriente, altera ab occidente [des aigles, l'un descendit du ciel depuis le levant, l'autre depuis le couchant].

Ce phénomène n'a rien d'étonnant dès lors que le verbe, qu'il soit placé avant ou après, réclame légitimement un nominatif [...].

Les Anciens s'autorisent néanmoins parfois à pré-poser un nominatif à la place du génitif pluriel, même si les verbes se rapportent au singulier aux éléments subséquents, c'est-à-dire aux éléments répartis, comme Homère, hoi de duo skopeloi ho men ouranon eurun hikanei [les deux écueils, l'un atteint le large ciel, od. 12, 73] :

alors que la grammaire exigeait d'employer le génitif, c'est-à-dire tôn de duo skopelôn [des deux écueils...], Homère a eu recours, à la place, au nominatif. De la même façon, si l'on dit

duo fratres, alter maior, alter minor est $\left[{ }^{*}\right.$ deux frères, l'un est le plus grand, l'autre le plus petit],

on fait une faute, car on doit dire

duorum fratrum, alter maior alter minor est [de deux frères, l'un est le plus grand, l'autre le plus petit],

et

duorum oculorum alter dexter, alter sinister est [de deux yeux, l'un est le droit, l'autre le gauche],

et non

duo oculi alter dexter, alter sinister est [*deux yeux, l'un est le droit, l'autre le gauche];

Tite-Live en XLI :

[QAN] periti religionum iurisque publici, quando duo ordinarii consules eius anni, alter morbo, alter ferro periisset, suffectum consulem negabant recte comitia habere posse [les spécialistes de questions religieuses et de droit public disaient que, puisque les deux consuls ordinaires de l'année, l'un était mort de maladie, l'autre d'un coup d'épée, le consul suffect ne pouvait valablement réunir les comices, 41, 18, 15-16] » (trad. Groupe Ars grammatica, 2010 : 107-109). phrase et ensuite décomposé en sujets divisés au singulier, on attendra un verbe au pluriel : si l'on veut que le verbe soit au singulier, la prolepse impose que le sujet global soit mis au génitif en fonction de complément des sujets divisés. Mais il y a des contreexemples, comme le montre le vers d'Homère ou la citation de Tite-Live.

Un problème apparaît alors pour les successeurs de Priscien : la figure désigne-t-elle la codification des accords dans le cas des structures complexes, ou les formules déviantes? à noter l'expression uitium facit, seul emploi de vitium au livre 17 de l'Ars Prisciani. Le vitium ne peut être excusatum que par l'autorité d'Homère ou de Tite-Live. 


\section{Syllepse, zeugma, synthèse}

C'est le « gros morceau », car il faut les traiter ensemble.

\subsection{Le zeugme}

Revenons à Donat [1]. Le zeugma faisait couple avec l'hypozeuxis: cette dernière, consistant en la répétition d'un élément, ne pose pas de problème d'accord et disparait sans encombre. Reste le zeugme: c'est la mise en commun d'éléments divers; si ces éléments sont des compléments comme dans TRO, il n'y a pas de problème, mais si ce sont des sujets ou des éléments associés dans un ablatif absolu, il y a problème. On voit bien la différence entre TRO et HIC ou encore :

SOC. Verg. Aen. 1, 553. Sociis et rege recepto

(litt. Nos compagnons et notre roi ayant été reçu).

Dans ces deux derniers exemples, on ne peut pas vraiment mettre en commun, car la forme serait différente (fuerunt dans un cas, receptis dans l'autre).

Mais se fait jour l'idée que le zeugme est un accord avec le plus proche : cette idée était préparée par Diomède qui met en avant le fait que l'élément mis en commun est à une place particulière (avant, au milieu, après) :

[7] Diomède, Ars grammatica, GL 1, 444.4-20

Zeugma est unius uerbi conclusio diuersis clausulis coniuncta, id est cum duo aut conplura ad unam partem orationis iungenda referuntur. Huius autem conclusionis diuersitas tribus locis posita indicatur. Fit enim aut cum praeponitur unum uerbum ad quod sequentes sensus conferantur, ut est uicit pudorem libido, timorem audacia, rationem amentia ; aut cum in medio sensuum loco positum reperitur, ut est : [TRO] Troiugena interpres diuum, qui numina Phoebi, / qui tripodas, Clari laurus, qui sidera sentis / et uolucrum linguas et praepetis omina pinnae :

sentis enim uerbum tam primis quam ultimis iungitur et ob id mesozeugma nominatur : aut certe cum uno uerbo sensus conplures cluduntur, ut est Tullianum : [NHT] nihilne te nocturnum praesidium [sg.] Palatii, nihil urbis uigiliae, nihil timor [sg.] populi, nihil concursus [sg. ou pl.] bonorum omnium, nihil hic munitissimus senatus habendi locus [sg.], nihil horum ora uultusque [pl.] mouerunt? conclusit enim uno uerbo mouerunt; unde hypozeugma dicitur.

Dans les exemples donnés, ce n'est pas forcément d'accord grammatical qu'il s'agit : dans le premier exemple, uicit convient sans problème avec chacun de ses sujets; dans TRO, on l'a dit, la nature des compléments n'a pas d'incidence sur la forme du verbe; pour NHT, c'est un peu plus compliqué, car le sujet est constitué par un mélange de termes au singulier et au pluriel, dont le dernier groupe, ora uultusque, est au pluriel.

De ce fait, certains exemples peuvent être sujets à une double analyse. Soit :

SVN. Verg. Bucoliques 1, 80-81.

Sunt nobis mitia poma [pl.], / Castaneae molles [pl.] et pressi copia lactis [sg.].

(litt. Sont à nous [Nous avons] des fruits savoureux, des châtaignes moelleuses et une quantité de fromage frais.)

Comme mouerunt dans NHT, le pluriel Sunt peut s'expliquer par la proximité de ses sujets au pluriel mitia poma (zeugme) ou par l'accord avec l'ensemble comprenant aussi le 
singulier copia (syllepse). Pour nous qui avons banalisé l'accord au pluriel, il n'y a pas lieu de voir là une quelconque figure.

\subsection{Concurrence et complémentarité zeugme/syllepse}

Du coup, certains exemples peuvent être traités soit par le zeugme, soit par la syllepse : pour faire simple, les humanistes hésitent entre zeugme et syllepse pour HIC, mais traitent massivement SOC, qui relevait de la syllepse pour les Anciens, par le zeugme.

Une nouvelle analyse se met en place : le zeugme désigne l'accord avec le plus proche, l'accord avec l'ensemble. Tel est le cas de Caesar et Cato equitant traité par Scaliger comme une conceptio (cf. supra), de même que Tu \& Lucina mihi cari estis, alors que Tu mihi carus est, \& Lucina l'est par la iugatio, terme latin équivalent du zeugma grec.

[8] Scaliger. De causis, 1540, livre 12, la figure, chap. 180, Iugatio (zeugme), p. 337.

Proxima huic 'iugatio' est, quam 'zeugma' græco vocabulo maluerunt appellare, cum tamen Latinis aliis uterentur. Nam quemadmodum in conceptione quod unius erat commune evadebat, sic in iugatione, quod unius est ita ad eum pertinet, ut eius significatui adiungat alterum. Per conceptionem sic loquare : tu et Lucina mihi cari estis; per iugationem sic : tu mihi carus es, et Lucina. Non igitur hic 'concipit', sed 'permittit' tantundem.

«Très voisine de la précédente est la iugatio (litt. couplage), qu'ils ont préféré appeler zeugma (zeugme) avec le vocable grec alors que pourtant, dans d'autres cas, ils faisaient usage de vocables latins. Car de même que, dans la conceptio (syllepse), ce qui concernait un seul terme se retrouvait commun, ainsi, dans la iugatio (zeugme), ce qui relève d'un seul touche celui-ci de telle sorte qu'à son signifié il en adjoint un autre. Par syllepse, tu peux dire : "Toi et Lucine m'êtes chers"; par zeugme: "Tu m'es cher, et Lucine <aussi>". Et donc, au lieu ici d'"englober" (concipere), on "relance" (permittere) d'une $<$ longueur> égale. »

La syllepse, qui avait tendance à englober toutes les constructions posant problème chez les Anciens, subit une tension entre une double tendance :

- extension à des constructions complètement banales... pour nous: SIT (cf. annexe), considéré comme syllepse par Linacre, Saturnius et Ramus, mais devenu un exemple type d'accord normal par exemple dans la Syntaxe latine d'Ernout et Thomas;

- restriction de son champ par concurrence avec le zeugme, comme on vient de le voir, mais aussi avec la nouvelle synthèse vient également empiéter sur son territoire.

\subsection{La synthèse}

34 Souvenons-nous de son sens chez Priscien : la sunemptôsis, c'est « la coïncidence possible de plusieurs sens possibles dans une même forme » (Baratin, 1989 : 448). Les médiévaux n'ont pas compris l'expression (sun «avec» + en «dans » + ptosis « cas »), rebaptisée synthesis, avec une étymologie rapprochant le terme de tithêmi, thesis, "(action de) poser ", d'où syn-thesis, « action de poser avec ».

Mais il est à noter qu'on trouve le terme latin correspondant, concidentia, chez P. Hélie dans une utilisation assez éloignée de celle de Priscien, dont M. Baratin donne l'explication suivante : 
La [gr.] sunemptôsis / concidentia, ou superposition de cas différents en une même forme, s'éclaire par un exemple cité au livre 18 (3, 347, 8-15), la construction grecque [gr.] pisteuein ha apèggelen (" avoir confiance dans ce qu'il a annoncé »), jugée problématique parce que pisteuein se construit avec le datif alors qu'ici [gr.] ha est un accusatif : l'interprétation proposée (haec synemptosis esse uidetur, ad uerbum enim sequens accusatiuo usus est) consiste à poser que dans la forme d'accusatif [gr.] ha, il y a superposition (" coïncidence ») du complément à l'accusatif de apèggelen et du datif complément de pisteuein. (Baratin 2011, p. 49-50)

[9] Pierre Hélie, Summa super Priscianum, éd. Reilly, 1993, p. 1005.64-67.

Concidentia vero est diversorum casuum vel numerorum vel generum vel personarum intransitiva constructio, ut [TVR] Turba ruunt, concidentia est quia diuersi numeri intransitiue construuntur ; ego ille, tu ille concidentia est quia diverse persone intransitive construuntur.

«La coïncidence est la construction intransitive de cas, de nombres, de genres ou de personnes divergents, comme [TVR] Une foule se précipitent : il y a coïncidence parce que des nombres divergents sont construits intransitivement ; moi celui-ci, toi celui-ci : il y a coïncidence parce que des personnes divergentes sont construites intransitivement. »

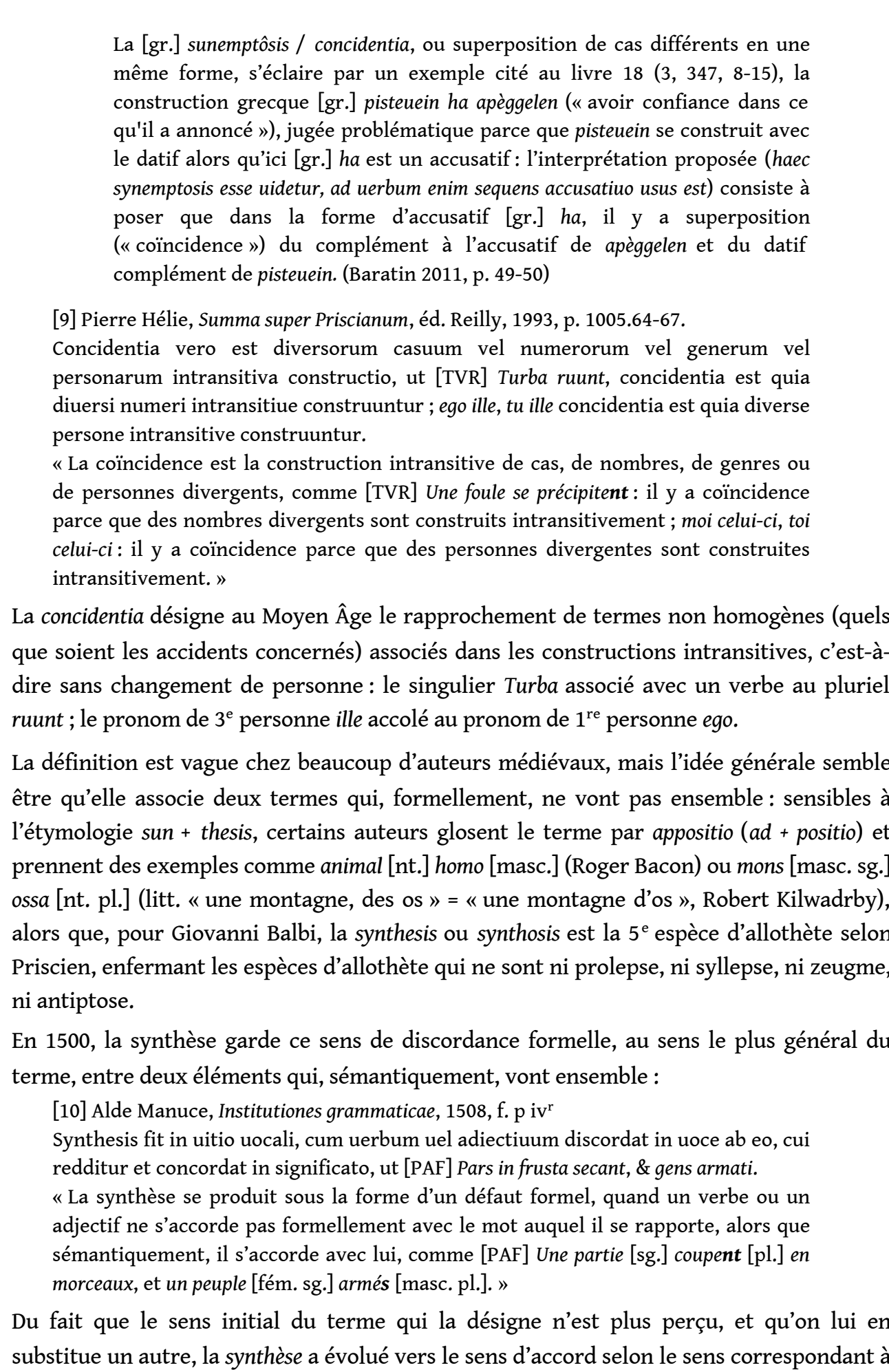
substitue un autre, la synthèse a évolué vers le sens d accord selon le sens correspondant à une distorsion au niveau du signifié. C'était venir empiéter sur le cas de la syllepse. Or il est tout à fait notable que le terme se soit maintenu, à la différence de allotheta: ainsi, deux siècles et demi plus tard, dans la première version de son article synthèse, Beauzée préfère le terme à syllepse, sans voir le problème que pose son origine ( $c f$.Colombat, 1993 : 368).

[11] Beauzée, art. « synthèse », Encyclopédie, tome 15, 1751, p. 764A (E.R.M.B.)

SYNTHÈSE, s. f. (Grammaire.) c'est une figure de construction que les Grammairiens appellent encore \& même plus communément syllepse : mais comme il y a un trope 
particulier qui a déja le nom de syllepse, \& qu'il peut être nuisible à la clarté de l'enseignement de désigner par le même nom des objets totalement différens, ainsi que je l'ai déja remarqué sous ce mot; je donne uniquement le nom de synthèse à la figure dont il est ici question.

« Elle sert, dit M. du Marsais, (FIGURE), lorsqu'au-lieu de construire les mots selon les regles ordinaires $\mathrm{du}$ nombre, des genres, des cas, on en fait la construction relativement à la pensée que l'on a dans l'esprit; en un mot... lorsqu'on fait la construction selon le sens, \& non pas selon les mots ».

\section{L'antiptose}

[12] Priscien, Ars, XVII, 160, GL 2, 187.12-188.2 :

Diuersi quoque casus uel casus pro casibus figurate tam a nostris quame a Graecis saepissime ponuntur, ut Vergilius [...] in I :

[VRQ] Vrbem quam statuo uestra est, subdicite naues

pro urbs quam statuo [...]

ut Terentius in Andria [...] :

[QAS] Quas credis esse has non sunt uerae nuptiae.

« Des cas différents également, ou des cas les uns à la place des autres, sont très fréquemment employés de façon figurée, aussi bien par les Latins que par les Grecs, comme Virgile en I :

[VRQ] Vrbem quam statuo uestra est, subdicite naues [La ville que je construis est la vôtre, tirez au sec vos navires].

pour urbs quam statuo,

comme Térence dans l'Andrienne :

[QAS] Quas credis esse has non sunt uerae nuptiae [ce n'est pas ce qu tu crois, de vraies noces]»

(trad. Groupe Ars grammatica, 2010 : 251-253).

L'antiptose traite de la substitution casuelle, et permet de traiter notamment de constructions difficiles du relatif dans lesquelles:

- soit l'antécédent au cas du relatif se trouve en dehors de la relative : VRQ;

- soit le relatif est inclus dans la relative, mais d'une nature telle qu'on doive l'en sortir et modifier son cas pour retrouver une phrase convenable: QAS.

bstitution casuelle est un cas assez clair pour permettre une extension à d'autres phénomènes de substitution, qu'on trouve par exemple dans le Doctrinal d'Alexandre de Villedieu :

[13] Alexandre de Villedieu, Doctrinal, vers 2599-2603

Pro numero numerum, pro casu ponere casum

te facit antitosis inter se dissona iungens.

saepius audiui tempus pro tempore poni :

ludere $=$ ludebat ad ludendumque uocabat ;

inque prophetiis mutantur tempora sacris.

«L'antiptose te fait mettre un nombre à la place d'un autre nombre, un cas à la place d'un autre cas, et unir des éléments qui discordent entre eux ; assez souvent j'ai entendu dire qu'on mettait un temps à la place d'un autre temps : jouer [inf.] pour il jouait et il appelait à jouer; et dans les textes sacrés des prophètes, il y a un échange des temps. »

À la Renaissance, l'antiptose est à nouveau restreinte à la substitution casuelle, et permet surtout de traiter les cas déjà évoqués de construction du relatif. Mais elle permet aussi à 
Nebrija d'expliquer l'exemple problématique de prolepse de Tite-Live QAN. Puisqu'il ne peut pas y avoir une prolepse bien construite, il y aura antiptose.

Dans cette extension large, elle sera relayée par l'énallage, substitution d'accidents, voire de parties du discours dont Linacre fait grand usage. Mais, d'action trop puissante (quelles sont les limites de son extension ?), l'énallage paraitra la panacée des mauvais grammairiens (les " grammatistes ») et la grammaire sanctienne l'éliminera, notamment au profit de l'hellénisme (Sanctius, Beauzée) ; il y a encore une entrée antiptose dans l' Encyclopédie, mais Du Marsais s'accorde avec Sanctius et Port-Royal pour y voir «une chimère et une absurdité ».

\section{Conclusion}

C'est l'histoire d'une dissolution, par dilution (pas complète) du système des figures de construction, dans un accord progressivement normalisé, très certainement en partie sous l'influence des structures vernaculaires correspondantes. Les figures sont toujours là, mais elles se chevauchent, ont une extension mal définie. Pratiquement toutes tournent autour du problème de l'accord, dont elles rendent compte pour des structures qui vont $\mathrm{du}$ (très) simple au complexe. Les grammairiens $\mathrm{du} \mathrm{XVI}^{\mathrm{e}}$ siècle reconstruiront le système en rétablissant le schéma quadripartite antique (adjonction, suppression, substitution, modification de l'ordre), dont la version la plus connue est peut-être celle de la Grammaire générale et raisonnée (II, 24)... Mais c'est une autre histoire.

Par ailleurs la figure de construction a été facilement transférée dans une langue non casuelle à ordre des mots contraints, comme le français. Mais le concept s'est alors considérablement simplifié: il ne comporte plus obligatoirement cette notion de discordance morphosyntaxique qui faisait l'essence même de la figure de construction en latin.

\section{BIBLIOGRAPHIE}

\section{Bibliographie secondaire succincte}

BARATIN, M. (1989) : La naissance de la syntaxe à Rome, Paris, Éd. Minuit.

- (2011) « Les figures de Priscien », in : M. Baratin M., C. Lévy, R. Utard \& A. Videau (éds), Stylus :

la parole dans ses formes. Hommage à Jacqueline Dangel, Paris, Classiques Garnier, p. 41-67.

colombat, B. (1993) : Les Figures de construction dans la syntaxe latine (1500-1780), Paris/Leuven, Peeters.

colombat, B. \& Rosier, I. (1990) : « L'allothète et les figures de construction dans le Catholicon de Iohannes Balbi : introduction, édition et traduction des chapitres sur les figures de construction ", Archives et documents de la SHESL, seconde série, 4, p. 69-161. 
GROUPE ARS GRAMMATICA (2010) : Priscien, Grammaire, livre XII - Syntaxe, 1, texte latin, traduction introduite et annotée, Paris, Vrin.

GRONDEUX, A. (2003) : « Turba ruunt (Ov. Her. 1, 88 ?) : histoire d'un exemple grammatical », Bulletin du Cange, 61, p. 175-222.

- (2013) : À l'école de Cassiodore. Les figures « extravagantes » dans la tradition occidentale, Turnhout, Brepols.

HOLTZ, L. (1981) : Donat et la tradition de l'enseignement grammatical, étude et édition critique, Paris, CNRS Éd.

ROSIER, I. (1988) : « Le traitement spéculatif des constructions figurées au XIII ${ }^{\mathrm{e}}$ siècle », in : I. Rosier (éd.), L'Héritage des grammairiens latins, de l'Antiquité aux Lumières, Paris/Leuven, Peeters, p. 181-204.

\section{ANNEXES}

\section{Les exemples (codés par ordre alphabétique)}

AQV. PRISCIEN XVII, 28

$\mathrm{AQVa}$ : Aquilae deuolauerunt, haec ab oriente, illa ab occidente ;

$\mathrm{AQVb}$ : Aquilarum altera deuolauit ab oriente, altera ab occidente.

*Aquilae altera deuolauit ab oriente, altera ab occidente.

(Les aigles s'abattirent en volant, l'un venant de l'orient, l'autre de l'occident ; Des aigles, l'un s'abattit en volant, venant de l'orient, l'autre de l'occident.

CON. VERG. Aen. 12, 161-169

Continuo [Interea] reges, ingenti mole Latinus quadriiugo uehitur curru [...] ; bigis it Turnus in albis [...] ; hinc pater Aeneas [...], et iuxta Ascanius, magnae spes altera Romae, procedunt castris.

(Immédiatement [Dans l'intervalle] les rois - Latinus à la taille puissante se déplace sur un quadrige [...], Turnus s'avance sur un bige aux chevaux blancs [...], de ce côté Énée le Père, et à ses côtés, Ascagne, l'autre espérance de la puissante Rome - sortent du camp.)

HIC. VERG. Aen. 1, 16-17

Hic illius arma, / Hic currus fuit.

(Là furent ses armes, là son char.)

ILI. OVIDE, Fastes, 4,54

Ilia [f. sg.] cum Lauso [masc. pl.] de Numitore sati [masc. pl.]

(litt. Ilia avec Lausus engendrés de Numitor)

NHT. CIC. Cat. 1, 1, 1

Nihilne te nocturnum praesidium Palatii, nihil urbis uigiliae, nihil timor populi, nihil concursus bonorum omnium, nihil hic munitissimus habendi senatus locus, nihil horum ora uultusque mouerunt?

(litt. En rien la garde nocturne du Palatin, en rien les vigiles de la ville, en rien la crainte du peuple, en rien le rassemblement de tous les gens de bien, en rien ce lieu si défendu pour les réunions du sénat, en rien les visages et les physionomies des présents ne t'ontils ému?) 
PAF. VERG. Aen. 1, 212

Pars in frusta secant

(litt. Une partie [sg.] coupent [pl.] en morceaux.)

QAN. TITE-LIVE 41, 18, 16

Quando duo ordinarii consules eius anni, alter morbo, alter ferro periisset...

(litt. Du fait que les deux consuls de cette année, l'un de maladie, l'autre au combat, était mort...)

QAS. TÉRENCE, Andrienne 47

Quas credis esse has non sunt uerae nuptiae.

(Ces noces que tu crois être des noces, ce ne sont pas de vraies noces)

REM. VERG. Aen. 1, 292-293

[Cana fides, et Vesta] Remo cum fratre Quirinus / Iura dabunt

([La blanche foi et Vesta,] Quirinus avec son frère Rémus donneront des lois.)

SIT. CIC. Fam. 14, 5, 1

Si tu et Tulia [...] ualetis, ego et suauissimus Cicero ualemus.

(Si toi et Tulia [...] vous allez bien, mon charmant Cicéron et moi-même nous allons bien.)

SOC. VERG. Aen. 1, 553

Sociis et rege recepto.

(litt. Nos compagnons et notre roi ayant été reçu.)

SVN. VERG. Bucoliques 1, 80-81

Sunt nobis mitia poma, / Castaneae molles et pressi copia lactis.

(litt. Sont à nous [Nous avons] des fruits savoureux, des châtaignes moelleuses et une quantité de fromage frais.)

TRO. VERG. Aen. 3, 359-361

Troiugena, interpres diuom, qui numina Phoebi, / qui tripodas, Clarii lauros, qui sidera sentis / et uolucrum linguas et praepetis omina pinnae [...]

(Fils de Troie, interprète des dieux, toi qui perçois les volontés de Phébus, le trépied prophétique, le laurier de l'hôte de Claros, les étoiles, le langage des oiseaux et les présages des volatiles rapides [...])

TVR. OVIDE Héroïdes 1,88 et 12, 143

Turba ruunt [Héroïdes, 1, 88 : Turba ruunt in me luxuriosi proci].

(litt. Une foule se précipitent / Une troupe, prétendants impudents, se précipitent sur moi).

VRQ. VERG. Aen. 1, 573

Vrbem quam statuo uestra est.

(La ville que je construis, c'est la vôtre.)

\section{NOTES}

1. Pour une identification plus facile, nous codons par les trois premières lettres les exemples qui sont répertoriés dans l'annexe.

2. Dans ce texte, nous préférons traduite verbum par « mot ", plutôt que par « verbe » (vs Baratin, 1989 : 289 et Holtz, $1981: 194$ définissant l'hypozeuxis). 


\section{RÉSUMÉS}

Cet article a pour objet d'étudier la création et le développement de la notion de figure de construction dans l'histoire de la grammaire latine. Il étudie le réseau des figures de construction à la Renaissance en montrant que leur origine remonte à la grammaire antique (Donat et Priscien notamment). Prolepse, syllepse, zeugme et synthèse fonctionnent en complémentarité pour rendre compte de constructions complexes ou jugées déviantes, par rapport à ce qui est reconnu comme un accord simple ou normal. La figure joue ainsi le rôle d'un outil proprement métalinguistique qui permet de dériver des structures complexes à partir d'énoncés simples, avant de se diluer dans les règles de la syntaxe d'accord.

The purpose of this article is to study the origin and development of the notion of figura constructionis in the history of Latin grammar. The paper examines the network of figures of syntax in the Renaissance and shows that their origin dates back to ancient grammars (Donatus and Priscian in particular). Prolepsis, syllepsis, zeugma and synthesis were used to account for complex or so-called deviant constructions in contrast with what is considered to be simple or normal agreement. Figures of syntax thus served as metalinguistic tools for the derivation of complex structures from simple sentences and clauses, before eventually being absorbed into the rules governing syntactic agreement.

\section{INDEX}

Mots-clés : figure de construction, syntaxe, accord, Latin, Antiquité, Moyen Age, Renaissance Keywords : figure of syntax, syntax, agreement, Antiquity, Middle Ages

\section{AUTEUR}

\section{BERNARD COLOMBAT}

HTL (UMR 7597), Université Paris Diderot 